Cerebrovasc Dis 2009;28:258

DOI: $\underline{10.1159 / 000228255}$

\section{Moyamoya Disease: Collateralization Is Everything}

Peter Vajkoczy

Department of Neurosurgery, Charité Universitätsmedizin

Berlin, Berlin, Germany

Moyamoya disease (MMD; moyamoya translates from Japanese as 'puff of smoke') represents a rare cerebral steno-occlusive disease characterized by the progressive occlusion of the terminal segments of the internal carotid artery and its branches. This process is accompanied by the development of an extensive basal collateral network of pathologic vessels [1]. The result of these stenoocclusions is a severe impairment of the cerebrovascular reserve capacity. Patients either present with obvious signs or symptoms, such as transient ischemic attacks or minor stroke, or with lessspecific subtle events, such as cognitive impairment, epilepsy, or chronic headache. Bilateral manifestation is common; however, unilateral occurrence is frequent in Caucasian populations in particular.

Whilst most cases have been reported in East Asia so far, both the number of pediatric as well as adult cases of MMD reported within the Caucasian population is growing steadily due to an increasing awareness of the disease. In fact, MMD is increasingly recognized in Europe as a cause of hemodynamic stroke and intracerebral hemorrhage in young patients.

Although the pathogenesis of MMD remains unclear, it is no longer a matter of discussion that both children and adults presenting with symptomatic MMD should be surgically revascularized $[1,2]$. Revascularization techniques are either direct or indirect. Direct revascularization strategies include 1 or 2 microanastomoses and a respective bypass using microsurgical techniques. The classic working horse of direct revascularization in chronic hemodynamic ischemia is the STA-MCA bypass. The advantage of direct revascularization is the immediate flow augmentation and flow replacement. In contrast, indirect revascularization procedures consist of surgical strategies where autologous tissue is placed on the brain surface (temporalis muscle, dura, galea, or omentum) and revascularization is achieved via angiogenesis/arteriogenesis from the graft into the leptomeningeal vascular system. Classic examples here are encephaloduroarteriosynangiosis (EDAS) or encephalomyosynangiosis (EMS). Our experience with more than 150 cases has taught us that MMD patients represent complex and high-risk surgical candidates due to the severe impairment of cerebral perfusion, the pathological changes in the vessel wall, as well as the necessity to revascularize often multiple vascular territories. Treatment decisions benefit from an established interdisciplinary infrastructure including experienced bypass surgeons, neurologists, neuroanesthesiologists and neurointensivists to provide an acceptably low complication rate.

MMD is a progressive cerebral steno-occlusive disease, as reflected by the current MMD classification proposed by Suzuki et al. [3]. Initially moderate stenoses in the basal arteries progress to high-grade stenoses and finally turn into occlusions with novel sites of steno-occlusive changes. This progression is slow and takes years, being compensated by the formation of thalamostriate and leptomeningeal collateral blood vessels [4], until hemodynamic compromise requires surgical revascularization.

While the natural history of MMD prior to surgery is well known, the relevance of progressive steno-occlusive changes following successful surgery is still uncertain. In the manuscript presented by Huang et al. [5] from the Division of Neurosurgery at the National Taiwan University, 23 patients with MMD, who underwent postsurgical revascularization of 38 hemispheres, were analyzed. The authors investigated the relevance of progressive steno-occlusive disease in their patients during a minimum of 3 years' follow-up, and could demonstrate progression of the vascular pathology in a relevant number of patients. Interestingly, they observed that progression of the steno-occlusive disease in the posterior cerebral artery occurred in approximately half of the patients within a short mean time of 18 months. In contrast to progression of stenoses of the internal carotid or middle cerebral arteries, these changes turned out to be prognostically unfavorable. One explanation for this may be that MMD patients are protected against changes in the anterior circulation by their bypasses (to the middle cerebral artery territory), but these remain insufficient to supply the posterior vascular territories in the absence of adequate collateralization from the posterior circulation. Furthermore, the authors demonstrated that successful MCA revascularization seemed to reduce the collateral blood supply via the posterior cerebral artery (mean time without surgery $>40$ months), with the consequence that MMD patients might loose leptomeningeal collateral blood flow and are at high risk of developing new neurological and ischemic deficits.

Thus, this manuscript addresses an interesting issue in MMD, i.e. the role of the posterior cerebral artery as a central source of supratentorial leptomeningeal collateralization. While progression of the internal cerebral artery stenoses does not seem to be of clinical relevance, due to the hemodynamic protection after surgical revascularization, the role of the posterior cerebral artery is causal and demonstrates the need for a detailed long-term followup of these patients. As a consequence, patients with progression in the posterior cerebral artery territory should be closely monitored and proactively revascularized at the occipital region as soon as angiographic changes become apparent.

\section{References}

1 Kuroda S, Houkin K: Moyamoya disease: current concepts and future perspectives. Lancet Neurol 2008;7:1056-1066.

2 Vajkoczy P: Revival of extra-intracranial bypass surgery. Curr Opin Neurol 2009;22:90-95.

- 3 Suzuki J, Takaku A, Kodama N, Sato S: An attempt to treat cerebrovascular 'moyamoya' disease in children. Childs Brain 1975;1:193-206.

-4 Czabanka M, Peña-Tapia P, Schubert GA, Woitzik J, Vajkoczy P, Schmiedek P: Characterization of cortical microvascularization in adult moyamoya disease. Stroke 2008;39:1703-1709.

$\checkmark 5$ Huang APH, Liu HM, Lai DM, Yang CC, Tsai YH, Wang KC, Yang SH, Kuo MF, Tu YK: Clinical significance of posterior circulation changes after revascularization in patients with moyamoya disease. Cerebrovasc Dis 2009;28:247-257.

\section{KARGER}

Fax +41613061234 E-Mail karger@karger.ch www.karger.com (c) 2009 S. Karger AG, Base

$1015-9770 / 09 / 0283-0258 \$ 26.00 / 0$

Accessible online at:

www.karger.com/ced
Peter Vajkoczy, MD

Department of Neurosurgery, Charité Universitätsmedizin Berlin

Campus Virchow-Klinikum

Augustenburger Platz 1, DE-13353 Berlin (Germany)

Tel. +49 30450560 002, Fax +4930450560 900, E-Mail peter.vajkoczy@charite.de 